\title{
Non-surgical minimally invasive rhinoplasty: tips and tricks from the perspective of a dermatologist
}

\author{
Ali Sahan ${ }^{1}$, Funda Tamer ${ }^{\text {凶 }}$
}

\begin{abstract}
Introduction: Rhinoplasty is one of the most common aesthetic procedures performed by plastic surgeons all over the world. In recent years, a non-surgical nose job has become the preferred option for most patients and specialists. Minimally invasive techniques already result in high satisfaction rates. Moreover, patients do not need anesthesia and there is no post-operative recovery period.

Methods: Between January 2016 and January 2017, 35 patients ( 33 female, 2 male) that had undergone nonsurgical rhinoplasty with dermal fillers were included in the study. Clinical and demographic features of the patients, our technique, satisfaction rates, and complications were discussed.

Results: We recorded an extremely high satisfaction rate and did not observe any complications. All of the patients returned to their normal daily activities immediately after the procedure.

Conclusion: Non-surgical nose augmentation with dermal fillers is an easy, safe, and comfortable technique. It appears that dermatologists need to assume a more active role in rhinoplasty.
\end{abstract}

Keywords: cosmetic techniques, dermal fillers, rhinoplasty

Received: 10 June 2017 | Returned for modification: 24 July 2017 | Accepted: 9 August 2017

\section{Introduction}

Non-surgical rhinoplasty with dermal fillers can be easily performed for most patients that have cosmetic concerns about the appearance of their nose. Proposed indications include patients that are not appropriate candidates for surgery or patients that do not want to have surgery and revision nose surgery. It is a fast, simple, safe, and cost-effective procedure without tissue damage. Therefore, non-surgical nose augmentation with dermal fillers is becoming more popular among specialists, including plastic surgeons. However, it is important to be familiar with the anatomical structure and the features of the fillers to attain successful results (1). Serious adverse effects including ophtalmic artery emboli following nasal augmentation with calcium hydroxylapatite filler, central retinal artery occlusion, visual acuity loss, and brain infarctions after nasal filler injection of hyaluronic acid (HA) have been reported $(2,3)$. Pressure necrosis of the nasal tip due to overinjection or ischemic necrosis of the nasal area due to arterial embolism should also be kept in mind (4). It has been suggested that skin necrosis of the nasal region is mainly caused by hydrophilic features of HA dermal fillers (5). Recently, Signorini et al. classified the filler complications as early reactions and late reactions. Early reactions include pain, ecchymosis, erythema, bruising, bleeding, vascular infarction, soft tissue necrosis, infection, hypersensitivity, inappropriate placement, and distant spread. Late reactions include inflammatory reactions such as granuloma, nodules, dyspigmentation, and displacement of fillers (6).

Cosmetic dermatologists perform cosmetic procedures, including facial filler injection. However, the glabella and nasal region are regarded as high-risk areas for skin necrosis and embolization. Therefore, many dermatologists avoid treating these areas. This article presents our experience of non-surgical rhinoplasty with dermal fillers by detailing our technique.

\section{Methods}

Medical records of 35 patients that had dermal filler rhinoplasty at the Al-Sa dermatology clinic between January 2016 and January 2017 were examined retrospectively. Photos were taken before and immediately after the injections. Prior nose surgery, coexistence of nasal obstruction or nasal deformity, previous cosmetic procedures, complications, and patient satisfaction were analyzed. All of the patients were followed for 6 months. Patient satisfaction was determined on a scale of o to 5 ( $0=$ dissatisfied, $5=$ very satisfied) (7).

We used 5\% lidocaine cream (Emla) for local anesthesia 30 minutes before the injection. We used a combination of $0.1 \%$ octenidine dihydrochloride and 2\% 2-phenoxyethanol (Octenisept $\left.{ }^{\circledR}\right)$ to disinfect the skin.

The procedure can be explained in two steps:

Step 1: A 22 gauge and $70 \mathrm{~mm}$ blunt cannula is used for filler injection. The entry point is made with a 21-gauge sharp needle on the tip of the nose. The cannula is slowly moved forward in the supraperiostal layer until it reaches the radix. The cannula is kept parallel to the nasal spine. The skin of the nasal dorsum is raised gently with the thumb and second finger during cannula movement. After the cannula reaches the radix, the filler is injected into the nasal dorsum in the supraperiostal layer using a retrograde linear threading technique. A 0.15 to $0.25 \mathrm{cc}$ volume of 15 $\mathrm{mg} / \mathrm{ml}$ HA dermal filler with $0.3 \%$ lidocaine (Juvéderm Volbella ${ }^{\circledR}$ ) is placed at point X and a 0.1 to 0.2 cc volume of $15 \mathrm{mg} / \mathrm{ml} \mathrm{HA}$ is placed at point Y. Point A is the nasal hump and point $Z$ is the nasal tip. The injection volume is 0.05 to $0.1 \mathrm{cc}$ for the nasal tip (Fig. 1). The cannula is removed following the tip injection. 
Step 2: Afterwards, the same cannula is inserted perpendicular to the nasal tip through the same entry hole. Then it is gently moved forward from the tip to the columella and nasolabial angle. After the cannula reaches the nasolabial angle, the filler is injected to point $\mathrm{P}$ and point $\mathrm{R}$ in the supracartilaginous layer using a retrograde linear threading technique. Herein, we used a $24 \mathrm{mg} / \mathrm{ml} \mathrm{HA}$ dermal filler with $0.3 \%$ lidocaine (Juvéderm Ultra $\left.4^{\circledR}\right)$. The injection volume is 0.1 to $0.15 \mathrm{cc}$ for point $\mathrm{P}$ and 0.1 to 0.2 cc for point R (Fig. 1). Finally, the cannula is removed from the tip of the nose.

The patients were advised not to wear glasses and to avoid exercise for a week after the rhinoplasty.

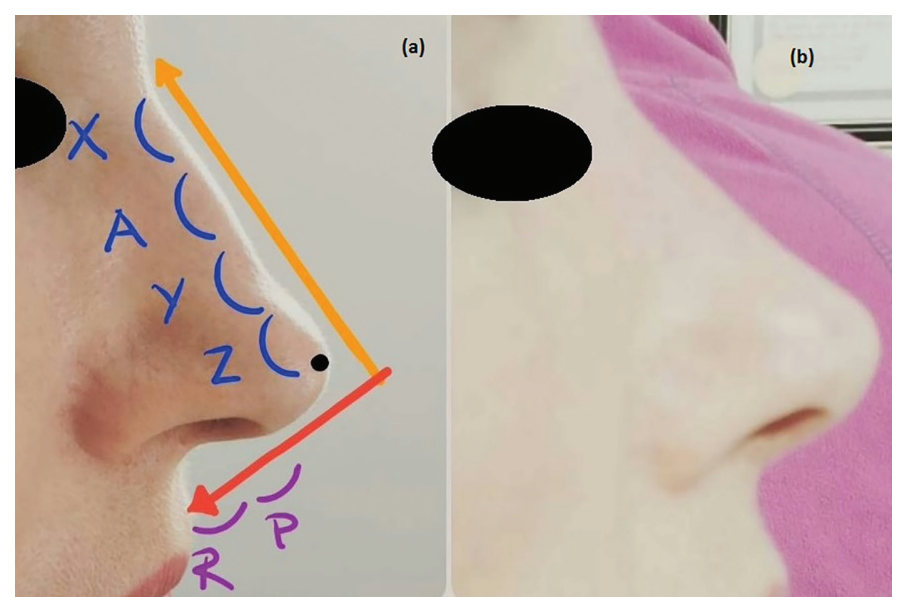

Figure 1 | Schematic view of filler rhinoplasty. The patient before (a) and immediately after (b) the procedure.

\section{Results}

Between January 2016 and January 2017, 35 patients (33 female, 2 male) were treated with non-surgical minimal invasive rhinoplasty at the Al-Sa dermatology clinic. The mean age of the patients was 27 (range: 20-35).

None of the patients had prior nose surgery or a non-surgical nose job, nasal obstruction, or nasal deformity. However, all of the patients had had prior botulinum toxin injections for facial wrinkles, and 13 patients (39.3\%) had had prior lip augmentation with HA fillers.

The patient satisfaction score was 5 for 32 patients; the satisfaction score was 4 for two patients and 3 for one patient. We sought to achieve the most natural appearance of the nose and the face (Figs. 2-4). However, three patients had very high and unrealistic expectations. Nevertheless, a very high patient satisfaction rate was noted in our study.

No complications were observed during the procedure or the 6-month follow-up. The patients were informed that the results could last approximately 8 months after the injection.

\section{Discussion}

Rhinoplasty is one of the most common cosmetic procedures performed by plastic surgeons. However, non-surgical nose jobs with a dermal filler are becoming increasingly popular in the world. Filler rhinoplasty has become an advantageous choice for patients that are afraid of surgery or general anesthesia. It is a fast, safe, simple, and effective method when compared with surgical rhinoplasty. Youn et al. evaluated the anthropometric analysis of 242 patients that had undergone HA filler rhinoplasty. Transient complications such as bruising, headache, swelling, and erythe- ma were reported in many patients. However, arterial occlusion was observed in only three patients. Needles were used instead of cannulas to perform filler injections in these patients. They suggested that filler rhinoplasty is as effective as surgical augmentation rhinoplasty based on anthropometric measures (8). Rho et al. used three-dimensional imaging to analyze facial anthropometric measures of 40 patients that had undergone filler rhinoplasty. Nasal volume increase, nose height increment, increase in nasolabial, nasofrontal, and nasomental angle, decrease in nose length, and nasofacial angle were observed. The results were similar to previously published studies including patients with surgical rhinoplasty (9).

Surgical rhinoplasty is associated with many complications, such as breathing difficulties as a result of reduced nasal airway size, hyposmia, numbness, infection and dislocation of implants, atrophy, skin necrosis, fibrosis, subcutaneous cysts, and granulomas. Moreover, serious post-operative deformities including pollybeak deformity characterized by a deep radix and prominent cartilaginous dorsum, asymmetries, deviations, overresection and irregularities of the nasal dorsum, alar collapse, slit-like nostrils, wide nasal tip or bridge, pinched tip, and overshortened nose have been reported. Therefore, 5 to $15 \%$ of patients undergo revision rhinoplasty following a primary surgical nose job (10).

Non-surgical rhinoplasty with fillers can be used to treat postsurgical rhinoplasty contour asymmetries. Bray et al. used Restylane-Lidocaine ${ }^{\circledR}$ to correct post-rhinoplasty complications such as right upper lateral cartilage disruption and tip scar. The filler was injected supraperiosteally with a 30-gauge needle using linear threading, serial puncture, and/or fanning techniques. They
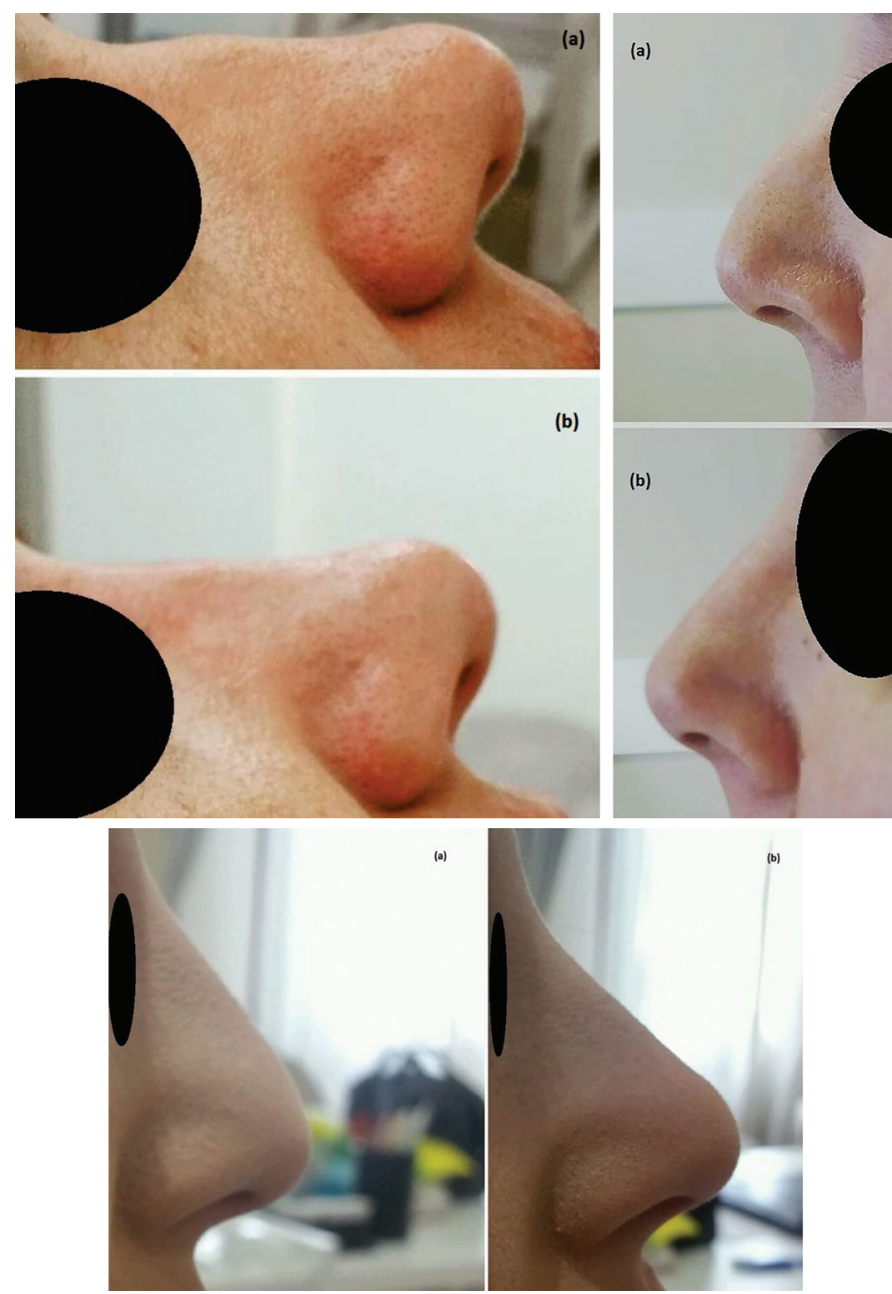

Figure $2 \mid$ Patients before (a) and immediately after (b) the procedure. 
reported slight complaints such as swelling, tenderness, and redness lasting for a few days (4).

HA fillers are biocompatible and biodegradable; however, complications such as foreign body reactions and delayed hypersensitivity can be observed with their injection. Moreover, Chae et al. reported the migration of injected HA filler from the nose to forehead, clinically presenting as two soft, skin-colored, subcutaneous nodules (11). Tissue necrosis is the most severe complication of fillers. One of the most dangerous area of the face for filler injection is the nose. The dorsal nasal arteries usually lie bilaterally to the nasal dorsum; however, they are not constant. Tansatit et al. classified dorsal nasal artery courses into five patterns; bilateral dorsal nasal arteries with unequal sizes, random distribution, a single dorsal nasal artery anastomosis with contralateral/ipsilateral lateral nasal artery, or bilateral lateral nasal arteries. They reported the nasal tip as a high-risk area, and they suggested that necrosis risk decreased at the upper sides of the nasal dorsum. However, patients with a single dorsal nasal artery had a high necrosis risk for all parts of the nose. Therefore, safety rules and using recommended techniques may not always prevent necrosis or ocular complications (12). Nevertheless, having full knowledge of the anatomical features of the nose, slow injection, avoiding excess pressure, using a small needle, and aspiration before injection minimizes the risk of complications $(6,13)$. We used a $15 \mathrm{mg} /$ $\mathrm{ml}$ HA dermal filler on the nasal dorsum of our patients to reduce the risk of vessel compression and pressure necrosis.

On the other hand, HA filler rhinoplasty can be completely reversed with hyaluronidase when needed. Signorini et al. recommend an injection of 10 to $20 \mathrm{U}$ hyaluronidase for areas less than $2.5 \mathrm{~mm}$ and two to four injections of 10 to $20 \mathrm{U}$ hyaluronidase for areas greater than $2.5 \mathrm{~mm}$ (6).

Appropriate patient selection is crucial for successful outcomes. Hence, all the patients should be examined carefully to decide on the procedure. Redaelli et al. recommended evaluating patients with five photographs taken from the front and the left and right lateral sides. The antero-posterior view of the nose, nose-tip and alar symmetry, and distances between the nose, eyes, and mouth should also be considered. They used fillers to correct the nasal spine starting at the root of the nose (13). Kim et al. reported that they began the procedure with filler injection at the columella-labial angle. Afterwards, augmentation was performed on the entire nasal dorsum from radix to tip. Then, additional filler was injected into the radix. Finally, small-volume boluses were injected into the nasal tip (14). Amore et al. described an Italian technique of modifying the nasal tip with fillers. The medial crura was pulled forward and down to subluxate the alar cartilage and stretch the fibrous septum. Approximately $0.22 \mathrm{ml}$ of HA was injected into the lower third of the space created. Then, 0.1 to $0.3 \mathrm{ml}$ of HA was injected deeply through the interdomal space (15).

People of different races have different facial features. For instance, Asians usually have a flat nose with a wide alar base, wide nasal tip, and retracted columella, whereas Caucasians have a prominent dorsum and nasal tip. Therefore, the procedure varies depending on patients' expectations (16). Here we report our experience with filler rhinoplasty in 35 Turkish patients and detail how we performed it. Understanding patients' expectations and explanation of the possible results of the procedure helped us achieve high patient satisfaction rates. We prefer to perform filler rhinoplasty on patients that have not had previous nose surgery to reduce certain risks, and there were no complications during the procedure and 6-month follow-up.

In conclusion, rhinoplasty with HA filler is an easy, quick, and cost-effective procedure when compared with surgical rhinoplasty. The procedure can be performed safely in dermatology outpatient clinics. Appropriate patient selection and a proper injection technique is crucial for reducing serious complications. We have shared the way we performed non-surgical rhinoplasty with our colleagues interested in cosmetic dermatology.

\section{References}

1. Kurkjian TJ, Ahmad J, Rohrich RJ. Soft-tissue fillers in rhinoplasty. Plast Reconstr Surg. 2014;133:121-6.

2. Cohen E, Yatziv Y, Leibovitch I, Kesler A, Cnaan RB, Klein A, et al. A case report of ophthalmic artery emboli secondary to calcium hydroxylapatite filler injection for nose augmentation: long-term outcome. BMC Ophthalmol. 2016;16:98.

3. Lin YC, Chen WC, Liao WC, Hsia TC. Central retinal artery occlusion and brain infarctions after nasal filler injection. QJM. 2015;108:731-2.

4. Bray D, Hopkins C, Roberts DN. Injection rhinoplasty: non-surgical nasal augmentation and correction of post-rhinoplasty contour asymmetries with hyaluronic acid: how we do it. Clin Otolaryngol. 2010;35:227-30.

5. Sung HM, Suh IS, Lee HB, Tak KS, Moon KM, Jung MS. Case reports of adiposederived stem cell therapy for nasal skin necrosis after filler injection. Arch Plast Surg. 2012;39:51-4.

6. Signorini M, Liew S, Sundaram H, De Boulle KL, Goodman GJ, Monheit G, et al. Global aesthetics consensus: avoidance and management of complications from hyaluronic acid fillers: evidence- and opinion-based review and consensus recommendations. Plast Reconstr Surg. 2016;137:961e-71e.

7. Bukhari AA. Non-surgical epicanthoplasty and rhinoplasty: epicanthorhinoplasty. JKAU: Med. Sci. 2010;17:3-9.

8. Youn SH, Seo KK. Filler rhinoplasty evaluated by anthropometric analysis. Dermatol Surg. 2016;42:1071-81.

9. Rho NK, Park JY, Youn CS, Lee SK, Kim HS. Early changes in facial profile following structured filler rhinoplasty: an anthropometric analysis using a 3-dimensional imaging system. Dermatol Surg. 2017;43:255-63.

10. Rettinger G. Risks and complications in rhinoplasty. GMS Curr Top Otorhi nolaryngol Head Neck Surg. 2007;6:Doco8.

11. Chae SY, Lee KC, Jang YH, Lee SJ, Kim DW, Lee WJ. A case of the migration of hyaluronic acid filler from nose to forehead occurring as two sequential soft lumps. Ann Dermatol. 2016;28:645-7.

12. Tansatit T, Apinuntrum P, Phetudom T. Facing the worst risk: confronting the dorsal nasal artery, implication for non-surgical procedures of nasal augmentation. Aesthetic Plast Surg. 2017;41:191-8.

13. Redaelli A, Limardo P. Minimally invasive procedures for nasal aesthetics. J Cutan Aesth Surg. 2012;5:115-20.

14. Kim P, Ahn JT. Structured nonsurgical Asian rhinoplasty. Aesthetic Plast Surg. 2012;36:698-703.

15. Amore R, Donnamaria D, Marini F, Amuso D, Leonardi V, Di Vito Francesco M. Nonsurgical rhinoplasty with injectable fillers: Italian technique for reshaping the tip of the nose. AJCS. 2015;32:172-7.

16. Liew S, Scamp T, de Maio M, Halstead M, Johnston N, Silberberg M, et al. Efficacy and safety of a hyaluronic acid filler to correct aesthetically detracting or deficient features of the Asian nose: a prospective, open-label, long-term study. Aesthet Surg J. 2016;36:760-72. 